\title{
Appearance of optical singularities at the light propagation through glasses with residual stresses
}

\author{
Krupych O., Vasylkiv Yu., Kvasnyuk O., Skab I. and Vlokh R. \\ Institute of Physical Optics, 23 Dragomanov St., 79005 Lviv, Ukraine, \\ e-mail: vlokh@ifo.lviv.ua
}

Received: 20.08 .2012

\begin{abstract}
We have shown that residual stresses existing in isotropic glass media lead to appearance of polarisation singularities of optical wave front. These singularities are characterised by the strength of topological defect equal to $\pm 1 / 2$ and should lead to the appearance of optical vortices with the topological charge \pm 1 . Annealing of the samples has led to annihilation of the polarisation singularities, homogenisation of spatial distribution of the phase difference and zeroing of the latter, thus suggesting disappearance of the residual stresses. The role of the singularities revealed in solving the known problem of 3D stress tensor field reconstruction is discussed.
\end{abstract}

Keywords: polarisation singularities, photoelastic tomography, stress tensor field reconstruction

PACS: $78.20 . \mathrm{hb}$, 42.50.Tx, 81.70.Tx, 07.10.Lw

UDC: 535.55

\section{Introduction}

Development of methods for reconstructing spatial distributions of internal stress fields in solid materials represents a hot topic in experimental mechanics [1]. The control of quality and durability of constructions requires nondestructive reconstruction of 3D stress tensor fields. One of commonly used methods for the reconstruction of stress tensor fields is based on a so-called 3D integrated photoelasticity [1,2]. This technique often demonstrates its efficiency while determining 3D stressed states in samples with high enough geometrical symmetry [2]. However, it cannot be efficiently applied when analysing the models for the most generally stressed states. On the other hand, reconstruction of $3 \mathrm{D}$ tensor stress fields should allow designing constructions and constructive elements characterised with maximal homogeneity, quality and mechanical durability, as well as minimal mechanical stresses.

As for optoelectronic elements, the problem concerns, first of all, fabrication of semiconductor and dielectric single crystals, glasses and heterostructures, which in the most of cases manifest some residual stresses and incompatible strains. A lot of theoretical approaches for solving the problems of integrated photoelasticity have been offered for the difficult case of asymmetrical objects. The principle idea of these approaches consists in consideration of the problem from the viewpoint of optical tensor field tomography [3-5]. However, these considerations too often represent no more than theoretical suggestions in need of further experimental verifications. Moreover, all of the above approaches cannot be implemented without additional a priori information about the stressed states. In addition, numerical methods for solving nonlinear equations necessarily appearing in the problem always lead to ambiguity of solutions.

Ukr. J. Phys. Opt. 2012, V13, №4 
A number of experimental methods have been proposed for determining three main parameters of the output optical beam, the characteristic phase shift $(2 \Delta)$, and the primary $(\theta)$ and secondary $(\gamma)$ characteristic directions [6,7]. All of these methods reveal relatively low accuracy, require long-term measuring procedures, and are laborious. The optical data obtained by means of these methods cannot be strictly used for reconstructing the stress fields. Furthermore, if the principal axes of the Fresnel ellipsoid are rotating while the optical beam propagates in a sample, the integral optical data obtained experimentally cannot in general be used for calculating stress distributions inside stressed bodies. In general, 3D photoelastic samples belong to the class of simultaneously spatially nonuniform and optically anisotropic (birefringent) media. An essential property of such media that makes them difficult for studies is rotation of their optical indicatrix around the light beam direction. Whenever the optical indicatrix rotation is present, all the indicatrix parameters obtained with standard polarimetric methods become integral and so, in general, it is impossible to derive the data about the stressed state at a given point inside a sample.

As a result, it is only possible to apply numerical calculations that rely upon the geometrical symmetry of the sample, the initial approximation about the stress field, and the integrated polarimetric data. This means that the principal problems of photoelastic methods for the stress tensor field reconstruction are a necessity of additional information about the stressed state and impracticability of obtaining data on the local state of optical impermeability tensor parameters, whenever the principle axis of the optical indicatrix rotates around the direction of light propagation.

Recently we have shown that the $2 \mathrm{D}$ stress states can be successfully reconstructed using the polarimetric techniques [8], whereas the 3D states can be reconstructed only if some information about the boundary conditions or the stressed state is available in advance [9]. Thus, the stress fields can be surely reconstructed if one finds the regions in the stressed samples where a $2 \mathrm{D}$ stress state exists or, at least, for which a 3D problem can be reduced to a $2 \mathrm{D}$ one.

We remind in this respect that $2 \mathrm{D}$ distributions of external fields usually induce optical singularities, which appear when the incident nearly plane-wave optical beams propagate through crystalline samples (see [10-15]). The optical vortices can be induced by the mechanical stresses appearing under torsion $[11,12]$ or bending $[13,15]$ of crystalline samples, or under compression of disks made of optical material along their diameters [14]. Notice that all of the stressed states mentioned above correspond to 2D stress distributions. Moreover, distributions of the stress tensor components which are needed for creating the vortices are known in advance for these stressed states. Then the first question that arises while solving the problem is as follows: do the polarisation singularities appear when the residual mechanical stresses are present in initially isotropic glass samples? The results of this work reveal the polarisation singularities in as-grown $\mathrm{Li}_{2} \mathrm{~B}_{4} \mathrm{O}_{7}$ and $\mathrm{LiB}_{3} \mathrm{O}_{5}$ glasses.

\section{Experimental procedure}

The platinum crucibles were used when preparing borate glasses. The glasses with the chemical formulae $\mathrm{Li}_{2} \mathrm{~B}_{4} \mathrm{O}_{7}$ and $\mathrm{LiB}_{3} \mathrm{O}_{5}$ were obtained. Their typical average sizes were $0.7 \times 4 \times 5 \mathrm{~cm}^{3}$. All the glasses were optically transparent, colourless, and of high enough optical quality. However, some spatial inhomogeneity of the refractive index was detected under visual examination of their optical transparency. In particular, this was peculiar for the $\mathrm{LiB}_{3} \mathrm{O}_{5}$ samples. These residual stresses were removed due to annealing of the samples at $680 \mathrm{~K}$. A more detailed description of the method used when preparing these glasses and of their main optical properties has been given in our recent work [16]. In our experimental studies we used a polarimetric setup described in detail in our work [11]. We utilised a wide, parallel circularly polarised incident beam while 
measuring the induced phase difference and placed the sample between crossed linear polarisers while measuring the optical indicatrix rotation angle.

\section{Results and discussion}

The appearance of our $\mathrm{Li}_{2} \mathrm{~B}_{4} \mathrm{O}_{7}$ glass sample placed between crossed polarisers is shown in Fig. 1. It is seen that annealing leads to lowering of the optical transmittance contrast observed for different regions of the sample. Obviously, this is caused by partial elimination of the residual stresses.
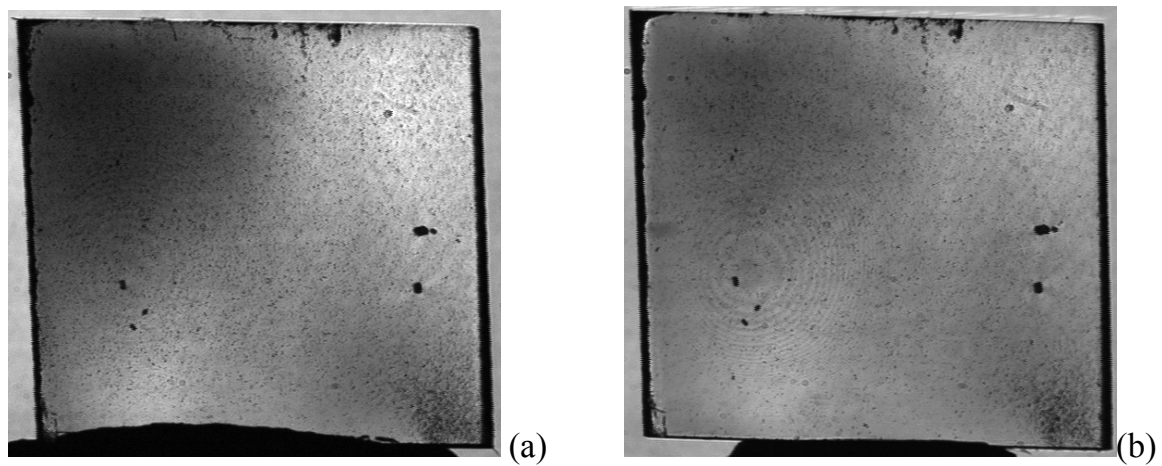

Fig. 1. Appearance of $\mathrm{Li}_{2} \mathrm{~B}_{4} \mathrm{O}_{7}$ glass sample placed between crossed polarisers as seen before (a) and after (b) annealing.

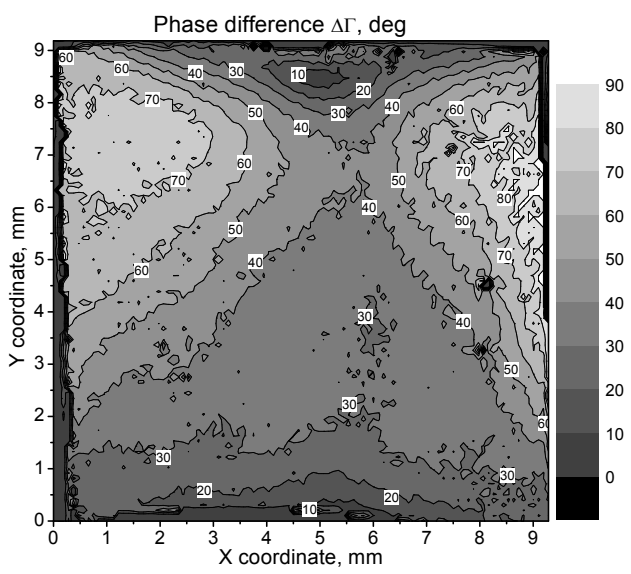

(a)
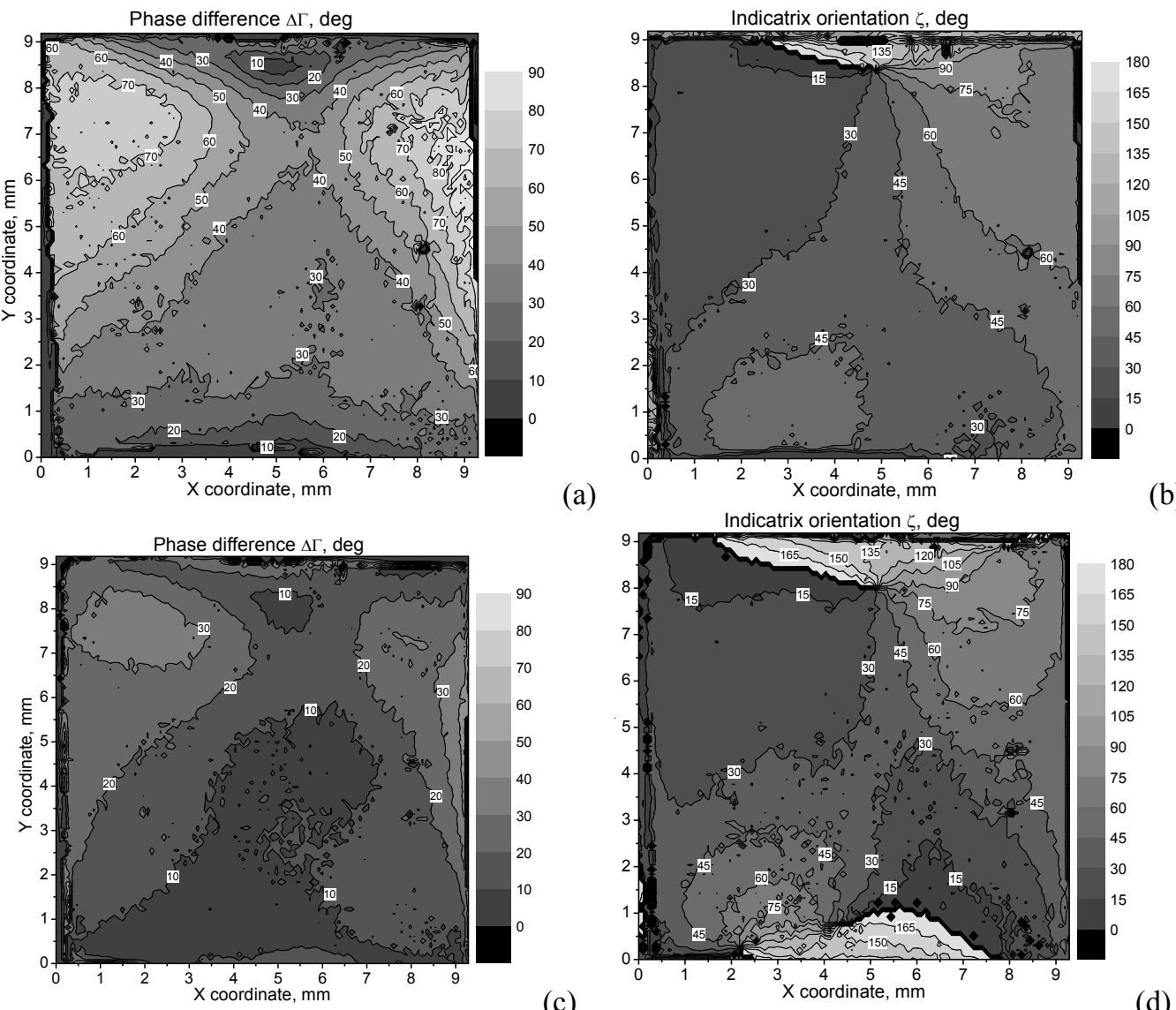

(b)

(c)

Fig. 2. The maps of phase difference $\Delta \Gamma(\mathrm{a}, \mathrm{c})$ and optical indicatrix rotation angle $\Delta \varphi(\mathrm{b}, \mathrm{d})$ obtained before $(a, b)$ and after annealing $(c, d)$ of $\mathrm{Li}_{2} \mathrm{~B}_{4} \mathrm{O}_{7}$ glass sample $(\lambda=632.8 \mathrm{~nm})$. 
Fig. 2a, $b$ show that both the phase difference and the optical indicatrix rotation for the asgrown sample of $\mathrm{Li}_{2} \mathrm{~B}_{4} \mathrm{O}_{7}$ glass reveal clearly expressed inhomogeneous distributions in the $X Y$ cross section, which are associated with specific spatial distributions of the residual stresses. Besides, one can see a singular point in the upper part of the map of optical indicatrix orientation (Fig. 2b). The coordinates of this singular point are the same as those of the zero phase difference point (see Fig. 2a). The anticlockwise direction of the optical indicatrix rotation around this polarisation singularity point should lead to appearance of an optical vortex with the topological charge equal to -1 . After annealing procedure, the two additional singularities appear in the bottom part of cross section of the sample (see Fig. 2b, c). These singularities are characterised by the opposite directions of optical indicatrix rotation, since the corresponding vortices should reveal the topological charges +1 and -1 . Quite probably, further annealing could have led to annihilation of these singularities, due to topological reaction and homogenisation of the phase difference and optical indicatrix orientation fields, which follow complete vanishing of the residual stresses.
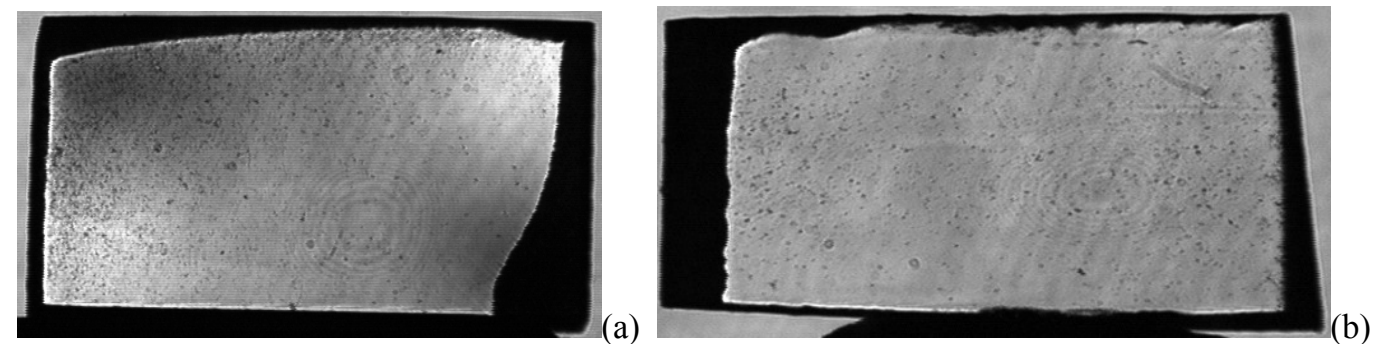

Fig. 3. Appearance of $\mathrm{LiB}_{3} \mathrm{O}_{5}$ glass sample placed between crossed polarisers as seen before (a) and after (b) annealing.

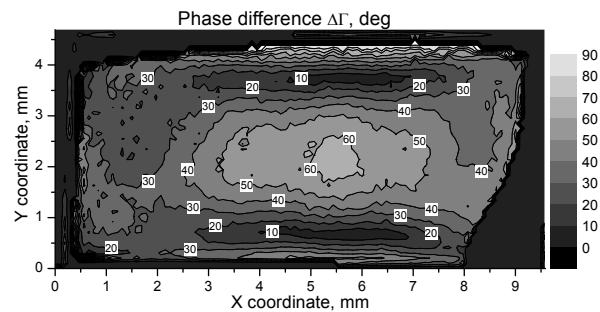

(a)
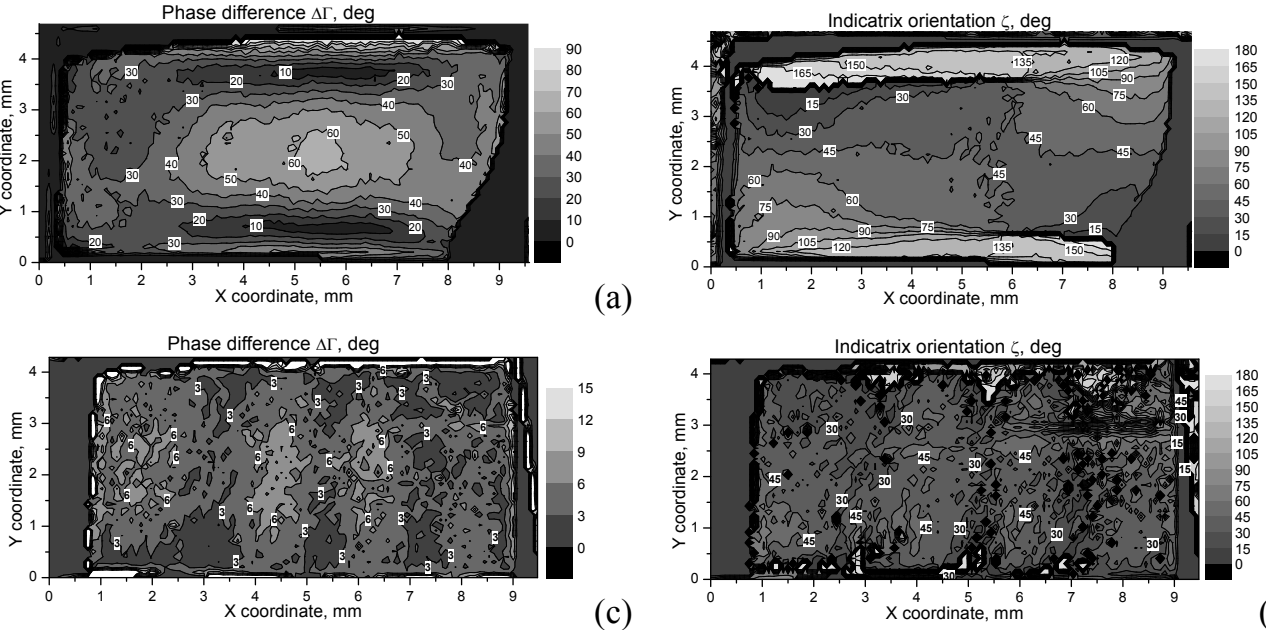

Fig. 4. The maps of phase difference $\Delta \Gamma(\mathrm{a}, \mathrm{c})$ and optical indicatrix rotation angle $\Delta \varphi(\mathrm{b}, \mathrm{d})$ obtained before $(a, b)$ and after annealing $(c, d)$ of $\mathrm{LiB}_{3} \mathrm{O}_{5}$ glass sample $(\lambda=632.8 \mathrm{~nm})$.

The similar situation is observed for our $\mathrm{LiB}_{3} \mathrm{O}_{5}$ glass sample (see Fig. 3 and Fig. 4). Two polarisation singularities are observed for the as-grown sample (Fig. 4a, b), which are characterised by the same topological charges $(-1)$ and a zero phase difference in the vortex cores. These polarisation singularities do not represent circles, being elongated along the $Y$ direction. This implies that the corresponding vortices should be elliptical, while the singularities of the 
phase front should conform to a mixed screw-edge dislocation. Unlike the case of the $\mathrm{Li}_{2} \mathrm{~B}_{4} \mathrm{O}_{7}$ glasses, annealing of the $\mathrm{LiB}_{3} \mathrm{O}_{5}$ sample has led to vanishing of the singularities. Indeed, that the retardation for the annealed sample is roughly equal to zero. Its residual value ( $\sim 6 \mathrm{deg})$ is quite small and almost homogeneously distributed over the $X Y$ cross section. The same is true for the optical indicatrix orientation peculiar for the annealed sample. Thus, the annealing has resulted in elimination of the residual stresses, being accompanied with disappearance of the polarisation singularities.

\section{Conclusions}

In the present work we have shown that the residual mechanical stresses existing in initially isotropic glass media yield in appearance of polarisation singularities of the optical wave front. The region in the vicinity of these singularities is, most probably, region of a $2 \mathrm{D}$ stressed state. The singularities are characterised by the strength of topological defects equal to $\pm 1 / 2$ and so they should lead to the appearance of optical vortices with the topological charge \pm 1 . The annealing of the samples brings about annihilation of the polarisation singularities, homogenisation of spatial distribution of the phase retardation and practical zeroing of the retardation, thus suggesting vanishing of the residual stresses.

Let us also make a final notice. Since the torsion stresses cannot induce polarisation singularities in non-crystalline isotropic media, it is probable that the singularities revealed by us in the glasses are induced by some stresses, which are peculiar for the bending stressed states. Then one can assume that a $2 \mathrm{D}$ distribution of the stress tensor components known for the region where the optical polarisation singularities appear can be used as the initial approximation in reconstructing 3D stress tensor fields in the whole sample.

\section{Acknowledgement}

The authors thank the co-workers of Crystal Growth Laboratory, the Institute of Physical Optics, for fabricating glass samples.

\section{References}

1. Aben H, Integrated photoelasticity, McGraw-Hill, New York, 1979.

2. Doyle J F and Danyluk H T, 1978. Integrated photoelasticity for axisymmetric problems. Exp. Mech. 18: 215-220.

3. Andrienko Y A, Dubovikov M S and Gladun A D, 1992. Optical tomography of a birefringent medium. J. Opt. Soc. Amer. A. 9: 1761-1764.

4. Andrienko Y A, Dubovikov M S and Gladun A D, 1992. Optical tensor field tomography: the Kerr effect and axisymmetric integrated photoelasticity. J. Opt. Soc. Amer. A. 9: 1765-1768.

5. Andrienko Y A and Dubovikov M S, 1994. Optical tomography of tensor fields: the general case. J. Opt. Soc. Amer. A. 11: 1628-1631.

6. Tomlinson R A and Patterson E A, 1998. Evaluating characteristic parameters in integrated photoelasticity. Proc. 11 ${ }^{\text {th }}$ Int. Conf. on Experimental Mechanics (Oxford, UK, 1998). Rotherdam: Balkema, 495-500 (1998).

7. Mangal S K and Ramesh K, 1999. Determination of characteristic parameters in integrated photoelasticity by phase-shifting technique. Opt. Las. Eng. 31: 263-278.

8. Vlokh R, Krupych O and Maksymuk O, 2003. Possible principles of optical 3D tensor stress field tomography. Ukr. J. Phys. Opt. 4: 41-47. 
9. Vasylkiv Yu, Kvasnyuk O, Krupych O, Mys O, Maksymuk O and Vlokh R, 2009. Reconstruction of 3D stress fields basing on piezooptic experiment. Ukr. J. Phys. Opt. 10: 22 37.

10. Skab I, Vasylkiv Yu, Smaga I and Vlokh I. 2011. Spin-to-orbital momentum conversion via electrooptic Pockels effect in crystals. Phys. Rev. A. 84: 043815.

11. Skab I, Vasylkiv Y, Savaryn V, and Vlokh R, 2011. Optical anisotropy induced by torsion stresses in $\mathrm{LiNbO}_{3}$ crystals: appearance of an optical vortex. J. Opt. Soc. Amer. A. 28: 633640.

12. Skab I, Vasylkiv Yu, Zapeka B, Savaryn V and Vlokh R, 2011. On the appearance of singularities of optical field under torsion of crystals containing three-fold symmetry axes. J. Opt. Soc. Amer. A. 28: 1331-1340.

13. Vlokh R, Pyatak Y and Skab I, 1992. Elasto-optic effect in $\mathrm{LiNbO}_{3}$ under the crystal bending. Ferroelectrics. 126: 239-242.

14. Skab I, Vasylkiv Yu, Krupych O, Savaryn V and Vlokh V, 2012. Generation of doubly charged vortex beam by concentrated loading of glass disks along their diameter. Appl. Opt. 51: $1631-1637$.

15. Skab I, Vasylkiv Yu and Vlokh R, 2012. Induction of optical vortex in the crystals subjected to bending stresses. Appl. Opt. 51: 5797-5805.

16. Adamiv V, Teslyuk I, Dyachok Ya, Romanyuk G, Krupych O, Mys O, Martynyuk-Lototska I, Burak Ya and Vlokh R, 2010. Synthesis and optical characterization of $\mathrm{LiKB}_{4} \mathrm{O}_{7}, \mathrm{Li}_{2} \mathrm{~B}_{6} \mathrm{O}_{10}$, and $\mathrm{LiCsB}_{6} \mathrm{O}_{10}$ glasses. Appl. Opt. 49: 5360-5365.

Krupych O., Vasylkiv Yu., Kvasnyuk O., Skab I. and Vlokh R., 2012. Appearance of optical singularities at the light propagation through glasses with residual stresses. Ukr.J.Phys.Opt. 13: $171-176$.

Анотація. У роботі показано, щзо залишкові напруження, які існують в ізотропних скляних середовищах приводять до виникнення поляризаційних сингулярностей оптичного хвильового фронту. Ці сингулярності характеризуються топологічними дефектами 3 силою, рівною $\pm 1 / 2$ і повинні приводити до виникнення оптичних вихорів з топологічним зарядом \pm 1 . Температурний відпал зразків приводить до анігіляції поляризаційних сингулярностей, гомогенізації розподілу різниці фаз $і$ ї̈ занулення, щчо свідчить про зникнення залишкових напружень. Обговорюється роль сингулярностей оптичного поля при вирішенні проблеми реконструкиії $3 D$ тензорного поля напружень. 